Published in final edited form as:

Chem Commun (Camb). 2018 October 28; 54(84): 11949-11952. doi:10.1039/c8cc05011b.

\title{
Sterol-modified PEG lipids: Alteration of the bilayer anchoring moiety has an unexpected effect on liposome circulation $\dagger$
}

\author{
Aaron Dolor $^{\mathrm{a}}$, Paul Kierstead ${ }^{\mathrm{b}}$, Zhipeng Dai ${ }^{\mathrm{c}, \mathrm{d}}$, Francis C. Szoka Jr. ${ }^{\mathrm{a}}$ \\ aPharmaceutical Sciences and Pharmacogenomics Graduate Program, Department of \\ Bioengineering and Therapeutic Sciences, University of California, San Francisco, San Francisco, \\ 94143 \\ bTricida Inc, South, San Francisco, California \\ 'ZoneOne Pharma Inc., San Francisco, California \\ ${ }^{d}$ Department of Bioengineering and Therapeutic Sciences, University of California, San Francisco, \\ San Francisco, 94143
}

\begin{abstract}
We synthesized and characterized two novel sterol-anchored polyethylene glycols (PEG) as potential alternatives to conventional phosphatidylethanolamine-PEGs. Liposomes containing the dicholesterol anchored PEG at 5 mole percent exhibit canonical PEGgylated-liposome behaviors including retention of encapsulated small molecules, low serum protein adsorption, and reduced cellular uptake yet they do not exhibit long circulation.
\end{abstract}

\section{Graphical Abstract}

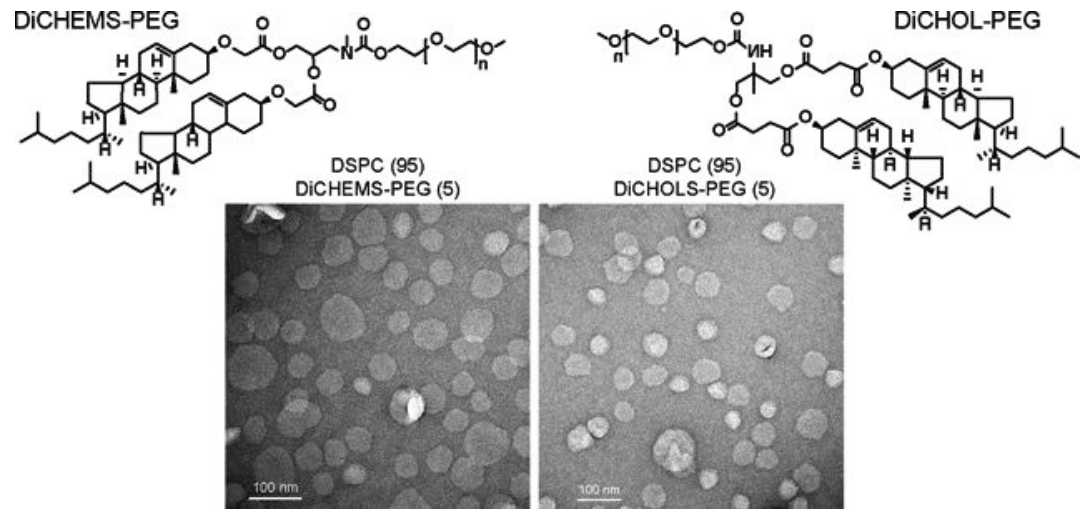

\footnotetext{
$\dagger$ Electronic Supplementary Information (ESI) available: Including experimental details, synthesis, and characterization.see DOI: 10.1039/x0xx00000x

Conflicts of interest

There are no conflicts to declare.

All animals were handled in accordance with guidelines established by the National Institutes of Health Guidelines for the Care and Use of Laboratory Animals, and with the approval of the Committee of Animal Research at the University of California, San Francisco, CA.
} 
Liposomes are the leading class of nanoparticle therapeutics. Low molecular weight drugs encapsulated in PEGylated liposomes exhibit an in vivo serum half-life of almost 2 days in humans and over 12 hours in mice. ${ }^{1,2}$ This is achieved through the incorporation of a 2000 $\mathrm{mW}$ polyethylene glycol (PEG) anchored to a 1,2-distearoyl glycerol-3-

phosphoethanolamine lipid (DSPE) which limits liposome aggregation, ${ }^{3}$ the adherence of serum proteins, and recognition by phagocytic cells. ${ }^{4}$

The pharmacokinetic benefits are dependent on DSPE-PEG lipids incorporating into the lipid bilayer. However, there are conflicting reports of the miscibility of DSPE-PEG when mixed with 1,2-distearoyl glycerol-3-phosphocholine (DSPC). In lipid-monolayer-coatedmicrobubbles DSPE-PEG2000-biotin is immiscible with DSPC as resolved by electron and fluorescence microscopy. ${ }^{5}$ In addition, Lozano et al. ${ }^{6}$ reported that DSPC and DSPEPEG2000 coexisted as a two-phase system and were almost completely immiscible in lipid monolayer mixtures. However, $\mathrm{Chou}$ and $\mathrm{Chu}^{7}$ reported that the two lipids were fully miscible in lipid monolayers and that addition of DSPE-PEG caused the monolayer to become more compressible. In addition, Dos Santos et al. ${ }^{8}$ showed that liposomes composed of DSPC and DSPE-PEG2000 exhibited similar pharmacokinetics in mice with or without cholesterol, which modulates interactions between DSPC and DSPE-PEG. ${ }^{6}$ This uncertainty in the behavior of DSPE-PEG200 in DSPC liposomes highlights the need for alternatives to DSPE-PEG2000 that interact more favorably with saturated lipids.

Cholesterol mixes favorably with $\operatorname{DSPC}^{9}$ and is commonly used in liposomes to improve bilayer stability. Previously, monolayers and liposomes prepared using sterol-modified lipids exhibited almost identical properties to liposomes prepared with conventional linear phospholipids and an equivalent mole ratio of cholesterol, ${ }^{10-13}$ which provides a rationale for the use of cholesterol as an anchor for PEG that would mix with bilayers composed of saturated phospholipids. Here, we present the biophysical properties of liposomes containing two different sterol-anchored PEGs and provide a preliminary assessment of their utility in liposomal drug delivery.

We synthesized two sterol-modified PEG-2000 (2000 kDa) molecules using standard approaches (Scheme 1). Glycerol-mimetic backbones were produced by reacting PEGnitrophenylcarbonate with 3-methylamino-1,2-propanediol or 2-methyl-2-amino-1,3propanediol, to generate compounds 3 and $\mathbf{5}$, respectively. PEG2K-2-methyl-2-amino-1,3propanediol was reacted with cholesteryl hemisuccinate through EDC coupling to yield a dicholesteryl hemisuccinate PEG (DiCHEMS-PEG) (6). For the other sterol-modified PEG, cholesterol was first derivatized using tert-butylbromoacetate in the presence of sodium hydride. The column purified product, $\mathbf{1}$, was deprotected using formic acid to yield compound $\mathbf{2}$, which was coupled to $\mathbf{3}$ in the presence of EDC to provide a dicholesterol PEG (DiCHOL-PEG) (4). DiCHEMS-PEG and DiCHOL-PEG were purified by dialysis with the DiCHOL-PEG requiring additional purification on a silica column.

To determine the fluidity of bilayers comprised of sterol-anchored PEGs and DSPC, liposomes were produced with increasing amounts of the dicholesteryl PEG lipids. Fluorescence anisotropy of 1,6-diphenyl-1,3,5-hexatriene (DPH) was measured in the various lipid compositions and the data plotted as the anisotropy normalized to the DPH 
probe in DSPC (Fig. 1). Pure DSPC undergoes a phase transition at $55^{\circ} \mathrm{C}$ marked by a dramatic reduction in anisotropy at higher temperatures (Fig. S13, ESI $\dagger$ ). The addition of increasing amounts of cholesterol eliminates this transition, flattening the DPH anisotropy versus temperature measurements (Fig. S13, ESI $\dagger$ ). Vesicles with DSPE-PEG in DSPC at 15 mole percent or greater exhibit an increase in the anisotropy (Fig. 1). This effect is due to the presence of DSPE-PEG micelles co-existing with DSPC-PEG in the bilayer (14). The anisotropy of DPH in vesicles containing DiCHOL-PEG or DiCHEMS-PEG with DSPC displayed behavior closer to cholesterol than DSPE-PEG (Fig. 1). The overall anisotropy of DPH is similar to that induced by cholesterol for both the sterol-anchored PEG molecules.

To compare the morphology of liposomes prepared using the sterol-anchored PEG molecules, transmission electron microscopy was performed. Formulations containing $5 \%$ DiCHEMS-PEG or DiCHOL-PEG with DSPC had vesicle profiles similar to DSPC:Cholesterol:DSPE-PEG formulations with $10 \%$ cholesterol (to match the total cholesterol present), and DSPC:Cholesterol:DSPE-PEG with the more conventional $40 \%$ cholesterol (Fig. 2).

Liposomes containing $5 \%$ DSPE-PEG, DiCHEMS-PEG, or DiCHOL-PEG were evaluated for bilayer stability by measuring the retention of encapsulated carboxyfluorescein (CF).

Liposomes loaded with $\mathrm{CF}$ were placed in serum and incubated at $37^{\circ} \mathrm{C}$. CF leakage was measured at various times over a week (Fig. 3). All liposomes exhibited an initial burst release of CF within the first $24 \mathrm{~h}$ before equilibrating in serum. DSPC liposomes containing $5 \%$ DiCHEMS-PEG or DiCHOL-PEG relea sed approximately $12 \%$ of encapsulated CF within 7 days which was comparable to the stable DSPC:Cholesterol (60:40) liposome formulation. Leakage was also comparable to DSPC:Cholesterol:DSPE-PEG (85:10:05) with $10 \%$ cholesterol.

To learn if the sterol-anchored PEG lipids could prevent or reduce plasma protein binding, we incubated DiCHOL-PEG and DiCHEMS-PEG and DSPC:Cholesterol:DSPE:PEG liposomes with bovine serum albumin (BSA) (Fig. 4). Liposomes were mixed with fluorescently labeled BSA and the amount of bound protein was determined following size exclusion chromatography to remove unassociated proteins. There were no significant differences among the four formulations investigated (Fig. 4). The trend in BSA binding to the various liposome compositions mirrored the binding trend for, mouse plasma proteins (Fig. S14, ESI $\dagger$ ).

Next, we measured cellular uptake in the presence and absence of apolipoprotein 3 (apoE3). ApoE3 can absorb onto the surface of neutral liposomes to facilitate hepatic clearance. ${ }^{15}$ Only the DiCHEMS-PEG showed an increase in uptake in the presence of apoE3 (Fig. 5, Fig. S15, ESI $\dagger$ ). In addition, the DiCHEMS-PEG had the greatest cellular association compared to all other liposome formulations.

$\dagger$ Electronic Supplementary Information (ESI) available: Including experimental details, synthesis, and characterization.see DOI: 10.1039/x0xx00000x 
Subsequently, liposomes of the four lipid compositions were produced with diameters that were within $\pm 10 \mathrm{~nm}$ and we measured the percent dose in mouse plasma 24 hours postdosing (Table 1). Unexpectedly, DSPC liposomes containing 5 mole percent DiCHEMSPEG or DiCHOL-PEG had the lowest percent dose remaining $24 \mathrm{~h}$ post dosing (Table 1). There was only approximately $3 \%$ of the dose remaining for liposomes with the sterolanchored PEGs, whereas DSPC:Cholesterol:DSPE-PEG (55:40:05) liposomes had 14\% of the dose remaining and DSPC:Cholesterol:DSPE-PEG (85:10:05) liposomes had $22 \%$ of the dose remaining at $24 \mathrm{~h}$ post dosing.

In summary, we synthesized and characterized two sterol-anchored PEG molecules with similar biophysical properties as potential alternatives to DSPE-PEG. Liposomes containing these dicholesteryl anchored PEGs at 5 mole percent exhibit an array of canonical PEGgylated-liposome behaviors including, retention of encapsulated small molecules, low serum protein adsorption, reduced cellular uptake yet they do not exhibit long circulation.

The primary structural difference between the two lipids is the use of cholesteryl hemisuccinate or cholesterol as the sterol anchor. Although cholesteryl hemisuccinate is conventionally used as a reactive form of cholesterol, ${ }^{16-18}$ it contains an ester linkage on the free hydroxy of cholesterol, where that ester carbonyl is believed to interfere with bilayer packing. ${ }^{12}$ Therefore, in the DiCHEMS-PEG formulation the cholesterol may be more accessible to protein adhesion. This structural difference could account for the increase in apoE3-driven uptake observed with the DiCHEMS-PEG lipid.

The two cholesterol modified lipids also differ in the aminopropyl moiety that anchors the cholesterol to the PEG. For the DiCHEMS-PEG, cholesteryl hemisuccinate was anchored to the 1- and 3-positions of the glycerol-like backbone with the PEG attached to the amine at the 2-position. Whereas in the DiCHOL-PEG, the cholesterol esters were attached to the 1and 2-positions with the PEG anchored to the methylamino group at the 3-position. It is not evident how these positional changes would influence the behavior of the appended 2000 MW PEG chain.

These sterol-anchored PEGs provide an interesting mystery. The properties of liposomes containing 5 mole percent of either sterol-anchored PEG or DSPE-PEG are similar but there is a substantial difference in the amount circulating $24 \mathrm{~h}$ post-injection in mice. In the case of the DiCHEMS-PEG, the sterol-anchor may facilitate the recognition of liposomes by lipophilic apoE proteins since they are known to interact with cholesterol, triglycerides, and lipoproteins. ${ }^{19,20}$ As a result, the apoE3 accumulation could increase the hepatic clearance of liposomes which might have contributed to the reduced circulation. Liposomes containing DiCHOL-PEG showed slightly greater uptake in cells than liposomes with DSPE-PEG which suggests that DiCHOL-PEG liposomes may also be cleared more rapidly due to increased cellular recognition.

Notably, the overall absorption of DiCHEMS-PEG or DiCHOL-PEG modified liposomes to cells in culture was relatively low since the adhesion of PEGylated liposomes to themselves or non-PEG-coated surfaces is greatly reduced. ${ }^{21}$ Thus, the complete explanation for the significantly lower levels in plasma remains puzzling. These results however, highlight the 
complexity of relationship between DSPE-PEG, DSPC, and cholesterol in lipid bilayers and raise questions regarding the criteria for the extended circulation of PEGylated systems Future studies with these lipids could be used to help clarify this complex relationship.

\section{Supplementary Material}

Refer to Web version on PubMed Central for supplementary material.

\section{Acknowledgments}

The authors would like to thank Dr. Charles Nobel and Dr. Mark Hayes of ZoneOne Pharma for their invaluable help and for fruitful discussions. We thank Dr. David-Huy Nguyen for help performing cellular uptake experiments. This work was supported by NIH T32 GM007175, the National Science Foundation Graduate Research Fellowship Program and generous financial assistance from Professor Jean Frechet to the Szoka laboratory.

\section{References}

1. Gabizon A, Catane R, Uziely B, Kaufman B, Safra T, Cohen R, Martin F, Huang A and Barenholz Y, Cancer Res., 1994, 54, 987-92. [PubMed: 8313389]

2. Drummond DC, Meyer O, Hong K, Kirpotin DB and Papahadjopoulos D, Pharmacol. Rev, 1999, 51, 691-743. [PubMed: 10581328]

3. Zalipsky S, Mullah N, Harding JA, Gittelman J, Guo L and DeFrees SA, Bioconjug. Chem, 1997, 8, 111-118. [PubMed: 9095350]

4. Immordino ML, Dosio F and Cattel L, Int. J. Nanomedicine, 2006, 1, 297-315. [PubMed: 17717971]

5. Borden MA, Martinez GV, Ricker J, Tsvetkova N, Longo M, Gillies RJ, Dayton PA and Ferrara KW, Langmuir, 2006, 22, 4291-4297. [PubMed: 16618177]

6. Lozano MM and Longo ML, Soft Matter, 2009, 5, 1822.

7. Chou T and Chu I, Colloids Surfaces B Biointerfaces, 2003, 27, 333-344.

8. Dos Santos N, Mayer LD, Abraham SA, Gallagher RC, Cox KAK, Tardi PG and Bally MB, Biochim. Biophys. Acta - Biomembr, 2002, 1561, 188-201.

9. Miyoshi T and Kato S, Langmuir, 2015, 31, 9086-9096. [PubMed: 26255826]

10. Kohli AG, Kieler-Ferguson HM, Chan D and Szoka FC, J. Control. Release, 2014, 176, 86-93. [PubMed: 24368300]

11. Huang Z, Jaafari MR and Szoka FC, Angew. Chemie Int. Ed, 2009, 48, 4146-4149.

12. Shaghaghi M, Keyvanloo A, Huang Z, Szoka FC and Thewalt JL, Langmuir, 2017, 33, 1440514413. [PubMed: 29120186]

13. Foglia F, Barlow DJ, Szoka FC, Huang Z, Rogers SE and Lawrence MJ, Langmuir, 2011, 27, 8275-8281. [PubMed: 21634402]

14. Sou K, Endo T, Takeoka S and Tsuchida E, Bioconjug. Chem, 2000, 11, 372-379. [PubMed: 10821653]

15. Yan X, Kuipers F, Havekes LM, Havinga R, Dontje B, Poelstra K, Scherphof GL and Kamps JAAM, Biochem. Biophys. Res. Commun, 2005, 328, 57-62. [PubMed: 15670750]

16. Venditto VJ, Watson DS, Motion M, Montefiori D and Szoka FC, Clin. Vaccine Immunol, 2013, 20, 39-45. [PubMed: 23114698]

17. Watson DS, Endsley AN and Huang L, Vaccine, 2012, 30, 2256-72. [PubMed: 22306376]

18. Huang Z and Szoka FC, J. Am. Chem. Soc, 2008, 130, 15702-15712. [PubMed: 18950160]

19. Akinc A, Querbes W, De S, Qin J, Frank-Kamenetsky M, Jayaprakash KN, Jayaraman M, Rajeev KG, Cantley WL, Dorkin JR, Butler JS, Qin L, Racie T, Sprague A, Fava E, Zeigerer A, Hope MJ, Zerial M, Sah DW, Fitzgerald K, Tracy MA, Manoharan M, Koteliansky V, De Fougerolles A and Maier MA, Mol. Ther, 2010, 18, 1357-1364. [PubMed: 20461061]

20. Cooper AD, J.Lipid Res, 1997, 38, 2173-2192. [PubMed: 9392416] 
21. Evans E, Klingenberg DJ, Rawicz W and Szoka F, Langmuir, 1996, 12, 3031-3037. 

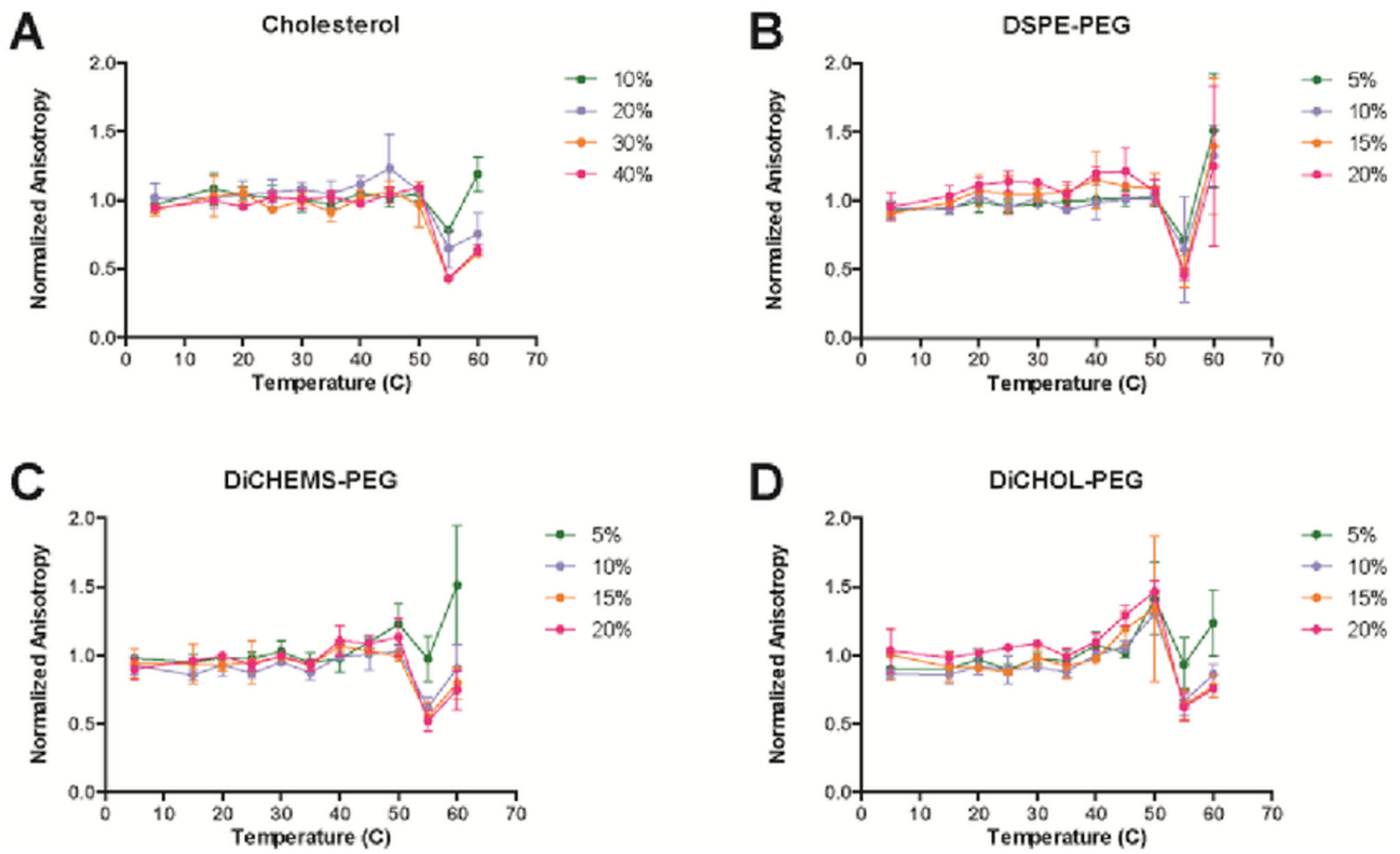

Fig. 1.

Normalized DPH fluorescence anisotropy of DSPC vesicles with increasing amounts of additives. A) Cholesterol, B) DSPE-PEG, C) DiCHEMS-PEG, and D) DiCHOL-PEG. DSPC liposomes containing sterol-anchored PEG exhibit similar anisotropy traces to liposomes with cholesterol. The mole percent of unmodified cholesterol is twice that of the mole percent of DiCHEMS-PEG or DiCHOL-PEG to have an equivalent amount of cholesterol in the bilayer. Errors bars represent mean \pm SD. 

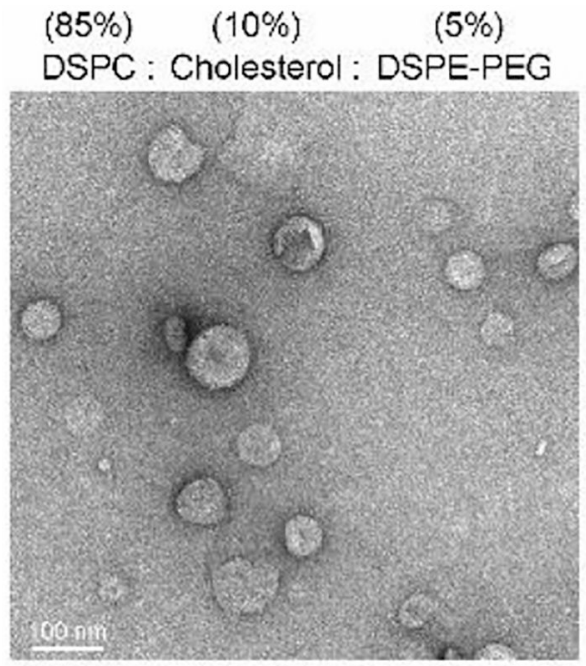

$(95 \%)$ DSPC : DiCHEMS-PEG

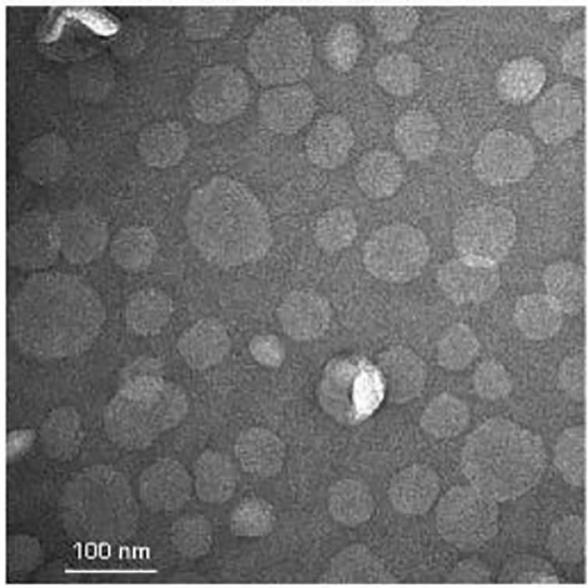

(55\%) (40\%)

$(5 \%)$

DSPC : Cholesterol : DSPE-PEG

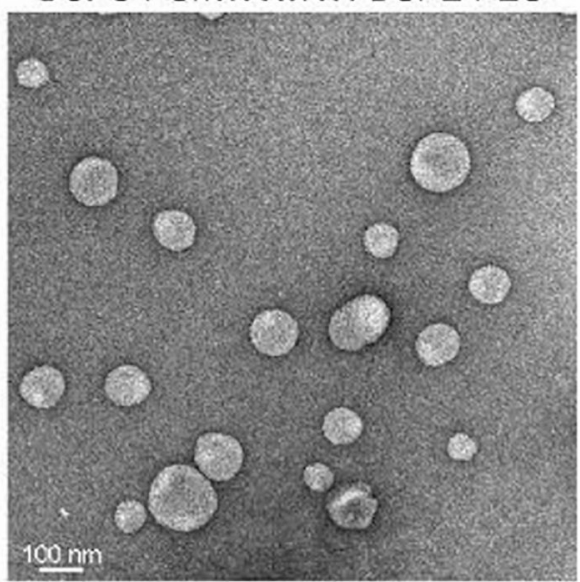

(95\%)

$(5 \%)$

DSPC : DiCHOL-PEG

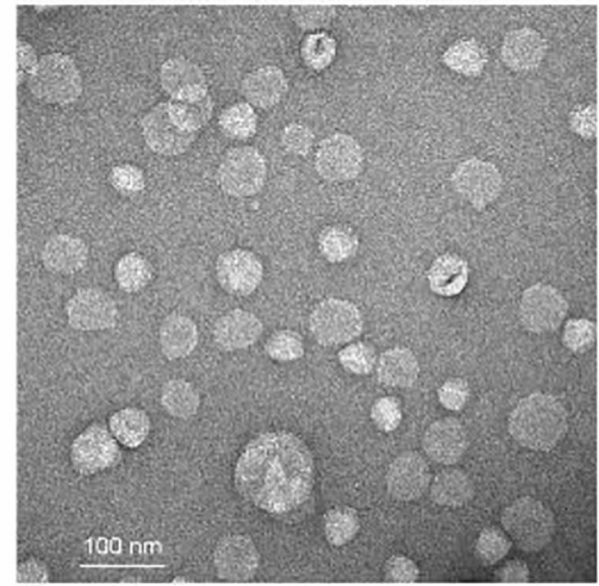

Fig. 2.

TEM images of liposome formulations. DiCHOL-PEG and DiCHEMS-PEG (bottom) form vesicles when mixed with DSPC comparable to vesicles formed from DSPC:Cholesterol:DSPE:PEG liposomes (top). 


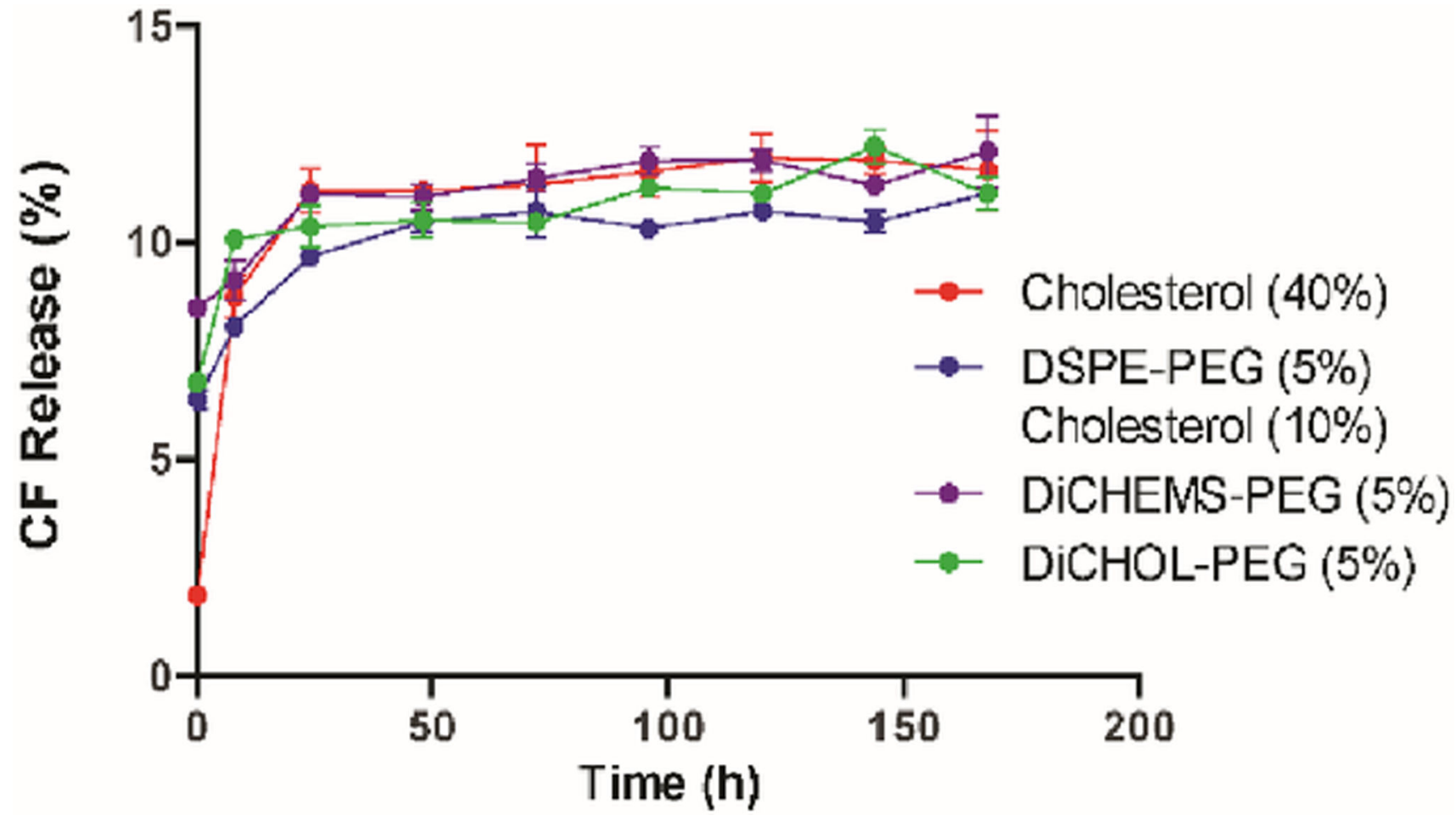

Fig. 3.

CF leakage from liposomes. DiCHEMS-PEG and DiCHOL-PEG liposomes exhibit similar $\mathrm{CF}$ leakage in serum as cholesterol containing liposomes. Error bars represent mean $\pm \mathrm{SD}$. 


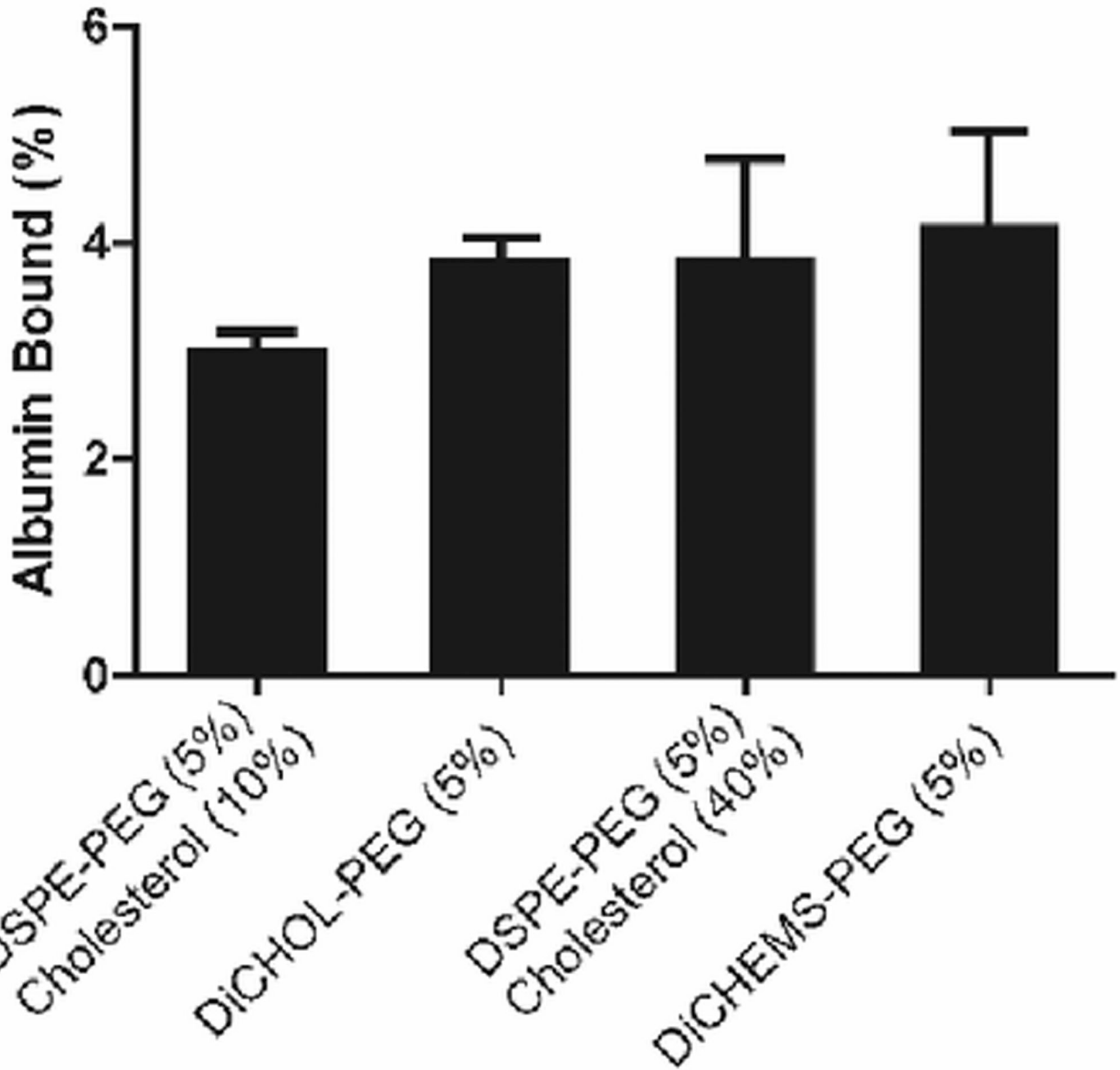

Fig. 4.

Normalized liposome protein binding. Liposome formulations exhibit minimal differences in non-specific protein binding. Percent BSA binding shows no significant changes in the levels of BSA associated with the liposomes. Protein content normalized to liposome concentration. Error bars represent mean $\pm \mathrm{SD}, \mathrm{n}=3$ /group. $p=0.64$ ANOVA. 


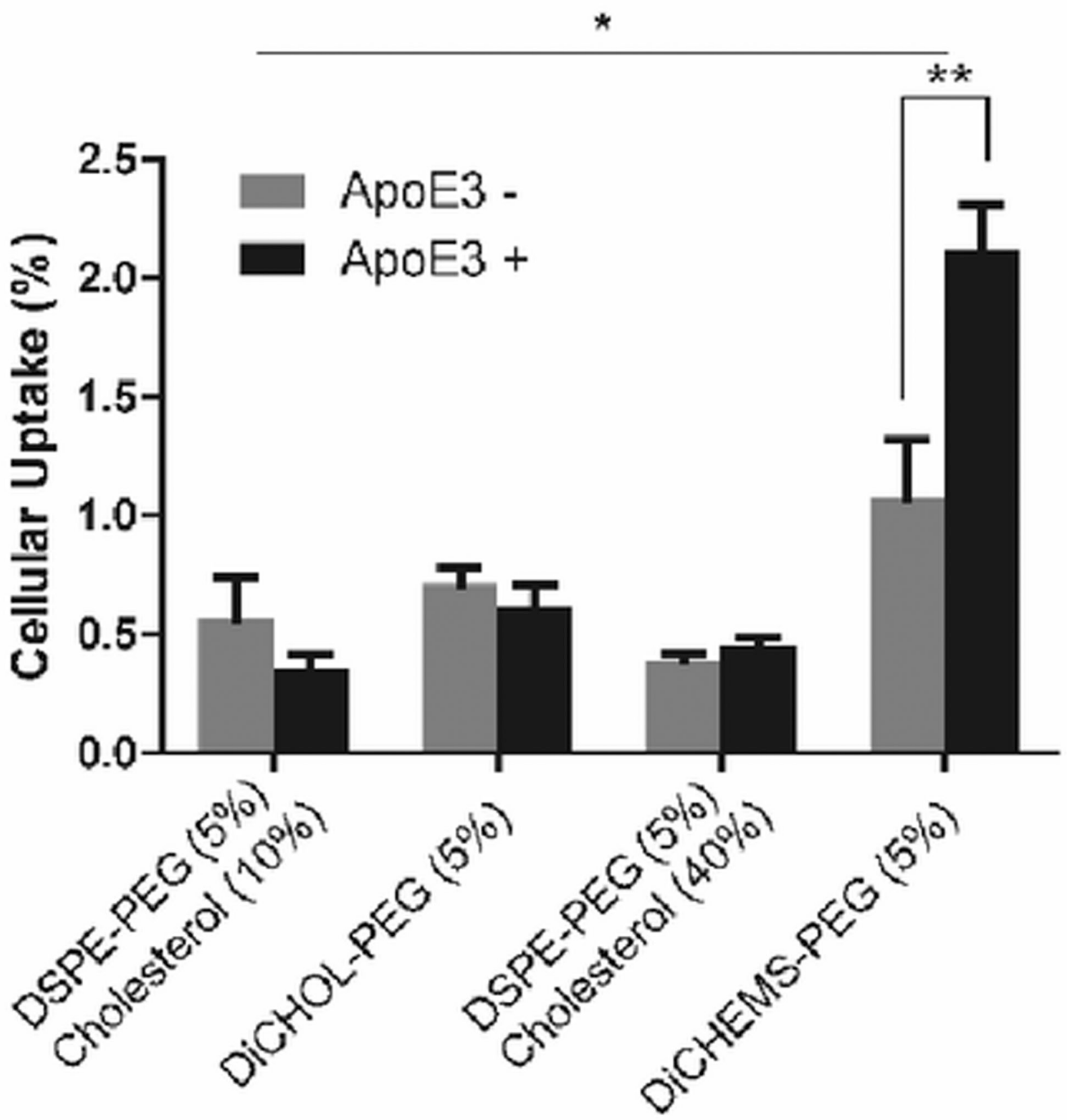

Fig. 5.

ApoE3 cellular uptake. Liposomes containing DiCHEMS-PEG exhibit increased cellular uptake in the presence of apoE3. Error bars represent mean $\pm \mathrm{SD}, \mathrm{n}=4$ wells/group. ${ }^{*} p<$ 0.05 ANOVA with Tukey's post-hoc test; $* * p<0.01, t$-test. 

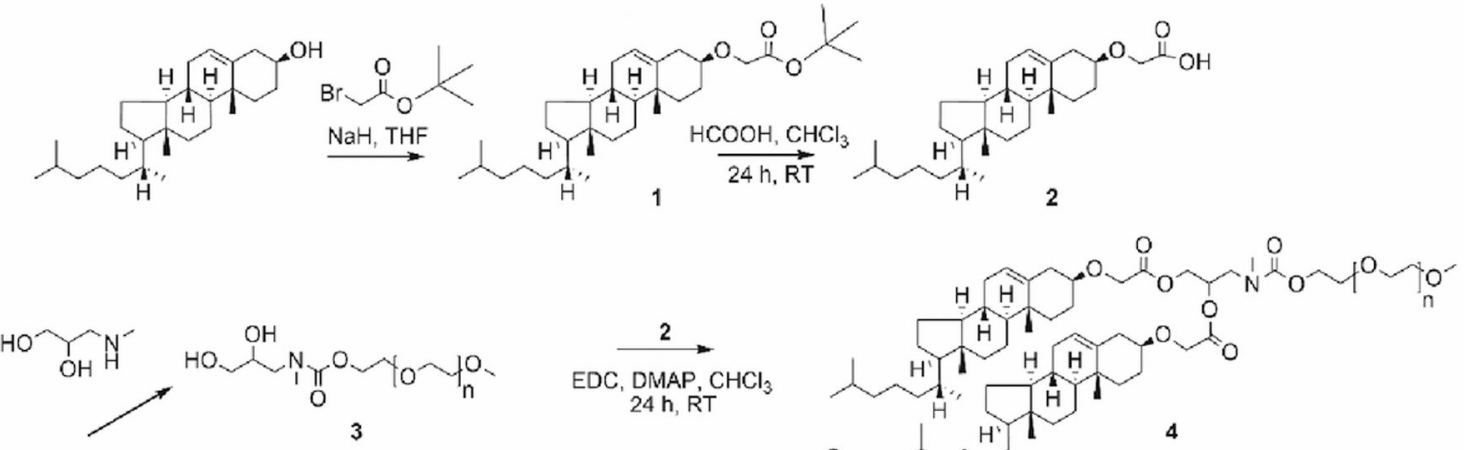

3 ON
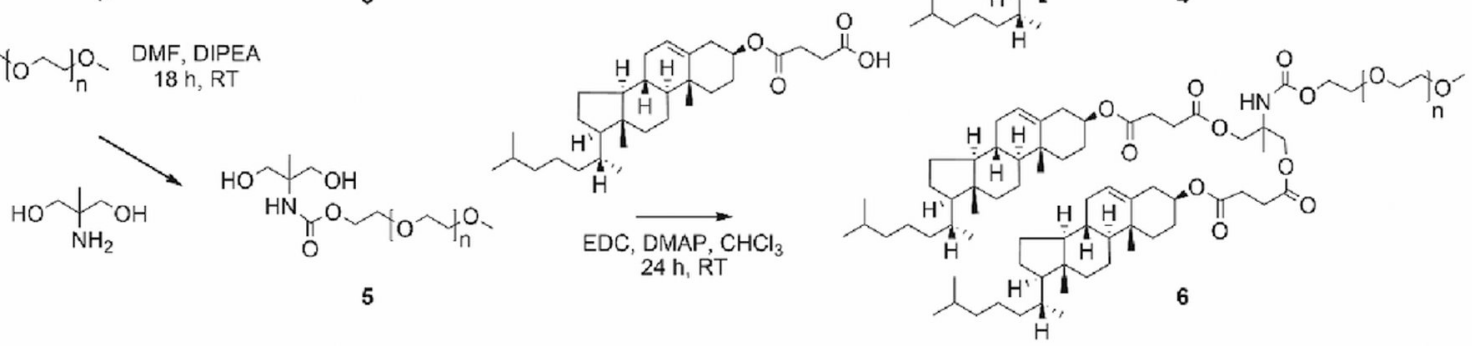

5

Scheme 1.

Synthetic scheme for synthesis of sterol-anchored PEG lipids.

Chem Commun (Camb). Author manuscript; available in PMC 2019 October 28. 


\section{Table 1}

Percent dose remaining of similar diameter DSPC vesicles containing DSPE-PEG with cholesterol, DiCHEMS-PEG or DiCHOL-PEG

\begin{tabular}{ccc}
\hline Formulation & Size & \% Dose at 24 h \\
\hline DSPC:Cholesterol:DSPE-PEG (55:40:05) & $100 \pm 3$ & $14 \pm 2.4$ \\
DSPC:Cholesterol:DSPE-PEG (85:10:05) & $94 \pm 4$ & $22 \pm 2.4$ \\
DSPC:DiCHOL-PEG (95:05) & $95 \pm 4$ & $3 \pm 0.2$ \\
DSPC:DiCHEMS-PEG (95:05) & $93 \pm 5$ & $2.4 \pm 1.2$
\end{tabular}

